\title{
A Survey on Semantic Sensor Web: Sensor Ontology, Mapping and Query
}

\author{
Xiang Wang, Xiaoming Zhang* and Mei Li \\ School of Information Science and Engineering, Hebei University of Science and \\ Technology, Shijiazhuang, 050018, China \\ wangxiang@hebust.edu.cn, zhangxiaom@hebust.edu.cn, limei@hebust.edu.cn
}

\begin{abstract}
Sensor Network has been widely used in all fields, and a large number of heterogeneous data is produced. In order to make these data shared and integrated semantically, semantic web technology is introduced in the sensor network. In this paper, we make a comprehensive survey about Semantic Sensor Web (SSW) from the perspective of ontology, mapping and query respectively. Firstly, we present state of the art of SSW from three aspects: ontologies before SSN ontology, SSN ontology and the extensions of SSN ontology, in which the extensions of SSN ontology are introduced emphatically. Secondly, we discuss the mapping from the stream data to ontology and focus on the comparison of the mapping languages in order to find out the best mapping method of SSW. Thirdly, we describe the research status of stream data query languages and analyze the characteristics, the application fields, the related researches and tools of the query languages in detail. Finally, the future research directions of SSW are proposed.
\end{abstract}

Keywords: Sensor network, Semantic Sensor Web, Sensor ontology, Mapping, Query

\section{Introduction}

In recent years, the sensor network is received more and more attention. It is increasingly deployed in all kinds of environments for different purposes. For example, In Environment-Monitor, urban traffic planning, flood prediction, health care, satellite sounding and other domains. It has been used in a wide range of applications. Therefore, mass sensor data can be produced continuously. However, the acquired sensor data lack of semantic information and may be heterogeneous in the syntax, schema and semantic level etc., and it's hard to be shared and integrated. If this issue can be solved, sensor network can has more application prospects [1].

In order to overcome the problem of lack of semantic in sensor network and manage a large number of heterogeneous data effectively, semantic web technology is introduced in the sensor network, which can describe information object, information sharing, information integration and knowledge reasoning. The added semantic can help to create the machine readable and self-describing data in the sensor network [1].The study of the sensor network must consider how to use semantic technology effectively.

Therefore, the combination of semantic web and sensor network becomes a hot research direction. Through the ontology technology, Semantic Sensor Web (SSW) can construct the shared conceptual model, enhance the sensor data semantics, and realize interaction and access of sensor data on the web. Semantic Sensor Web has the following characteristics:

(1) Sensor data is annotated with semantic to improve the interoperability of data [2].

(2) The context information such as time, space, theme for the sensor data is added.

(3) SSW combines multi-type sensor data to support intelligent decision synthetically.

(4) SSW can resolve the heterogeneous of sensor data to express the data uniformly. 
(5) The description capability, the sharing and reuse capability of the sensor data can be increased.

In this review, we investigate the related work of Semantic Sensor Web in recent years especially from the ontology, mapping and query perspective to show the state of the art and future research directions in this area. The development of sensor ontologies is introduced and the SSN ontology and the extensions of SSN ontology are discussed emphatically. Meanwhile, the comparison criteria for the ontologies are given. Mapping methods which from stream data to the ontology are analyzed and the mapping languages are compared. Semantic query methods of stream data based on ontology are discussed. The comparison of query languages is done according to the comparison criteria which are presented in this paper. Finally, the future research directions of Semantic Sensor Web are presented.

The reminder of this paper is organized as follows. Section 2 presents the problem description of this article. Section 3 reviews the state of the art of the sensor ontology. Section 4 describes the mapping from stream data to ontology. Section 5 introduces query languages of the sensor data, and compares their different. Section 6 gives the future research directions of the Semantic Sensor Web.

\section{The Problem Description}

Semantic Sensor Web has been a hot area of research for several years. W3C founded the "Semantic Sensor Web" incubator working group which aimed to create and define ontology knowledge of sensor and sensor network and formulate semantic markup language based on service applications of the sensor network [2].

Semantic Sensor Web is the combination of the semantic web technology and sensor network which uses declarative descriptions of sensors, networks and domain concepts to search, query and manage the network and sensor data. On the other hand, it is an OGC-style sensor web enriched with semantic annotation, querying and inference [2]. In this paper, we present architecture of Semantic Sensor Web which uses three layers of semantics and technology to provide services, and it is showed in Figure 1. They are the sensor data source layer, the data integration layer and the application layer. The contents of the three layers are introduced in details in the fallowing.

\subsection{The Sensor Data Source Layer}

The bottom layer is the data source layer of the sensor network. There are a large number of sensor data in the sensor network, and the sensor data are stream data which are the infinite timestamp tuple sequence. The timestamp character of the sensor stream data which indicates when the tuple occur is unique. Therefore, the following research content in this review must consider this issue.

Sensor data has two representational forms: One is the data which is stored in the relational stream database, and another is the stream data which are collected in real time. The different data of representational forms is processed by different method when these data are integrated.

\subsection{The Data Integration Layer}

In data integration layer, the sensor data are represented, managed and queried. There are three contents in this layer: ontology, mapping and query. The sensor ontology can capture the domain knowledge and provide the common understanding of it. It can provide a kind of shared vocabulary and concepts existed in the sensor network areas and their properties along with the mutual relations. The sensor ontology can provide critical support technology for the sensor network, and improve the semantic interoperability and 
integration. Because sensor data has time character, in the sensor ontology, time character must be considered [3].

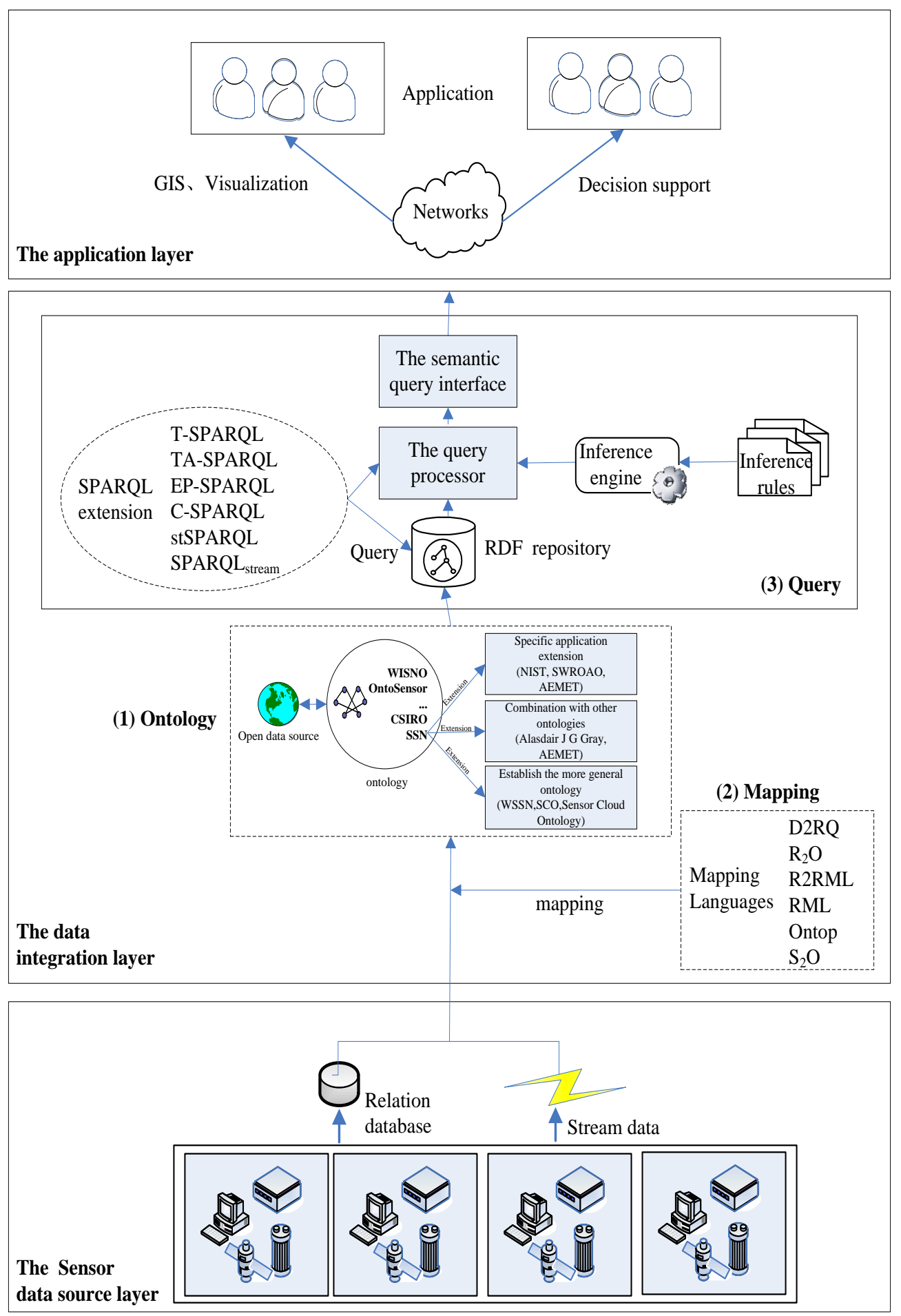

Figure 1. The Architecture of Semantic Sensor Web

Sensor ontology plays an important role in Semantic Sensor Web. Its roles can be summarized as follows:

(1) It establishes the uniform concept system for different sensor network fields and does not depend on a specific application. 
(2) It can connect the sensor data with other open data sources to realize the link of knowledge in order to give a wider range of application to users.

(3) It solves the time character of sensor data well, and records timestamp in detail;

(4) It implements the knowledge reasoning of the sensors and provides good decision support for perceiving the objective world.

Before SSN ontology [4], there are some sensor ontologies which are developed for the specific application. SSN ontology is an important general sensor ontology which focuses on many existing issues. In recent years, many extension ontologies which based on SSN ontology have appeared. Some of them extended SSN for the specific application, some focus on working together with other ontologies, and some served for establishing a more general ontology. Whatever extensions, these ontologies give a unified model for the sensor data, so that human with machines or machines can communicate with each other.

With the sensor ontology model, the mapping model between the stream data and ontology must be established. Some mapping languages which establish a mapping relation between the stream data and the sensor ontology can implement the mapping process. The mapping relation can help to realize the semantic query transformation or the transformation from the sensor data to the instance data. The mapping processor can do that.

The purpose of the mapping is query, and query can get information and meet the needs of users. Query languages can provide query statements which are Interpreted and implemented by the query processor. In the query process, the inference rules are established which are realized by the inference engine. Several mapping languages have the corresponding query processor, and we can use these processors or build our own query processor.

\subsection{The Application Layer}

The purpose of the query is for applications which can be realized by the semantic query user interfaces. Semantic Sensor Web has wide applications, for example, in Geographic Information System, visualization, decision support, and environmental monitor, etc. In the future, there are several application directions, for example, Semantic Sensor Web can research in the cloud platform, link with the open data source and combine with social network and artificial intelligence, etc.

\section{The State of the Art of the Sensor Ontology}

Before SSN ontology, a lot of sensor ontologies have appeared, and they played an important role in their own specific field. Compton, et al., [3] reviewed the typical sensor ontologies and analyzed their concept scope, expressive ability and reasoning ability. In 2009, the W3C working group developed SSN ontology. SSN ontology is a general sensor ontology which provides a unified standard to describe the sensor network data. In recent years, many ontologies which are the extensions the SSN ontology appeared, and they expand the application range. The development process of sensor ontology is shown in Figure 2. In the following, we introduce the sensor ontologies in details from three stages.

\subsection{Ontologies before SSN Ontology}

Before SSN ontology, a lot of sensor ontologies have been developed. These ontologies are mainly divided into two categories: the ontologies of specific application, and the general ontologies of the sensor network.

The ontologies of specific application are CESN [5], A3ME [6], SWAMO [7], MMI [8], OOSTethys [9] and ISTAR [10] etc. 
The CESN ontology is developed in the Coastal Environmental Sensing Networks project for sensor networks for coastal observing. The MMI ontology and the OOSTethys ontology were intended for marine equipment interoperability and focus on the system structure and the observation process and results. The A3ME ontology was developed for classifying the heterogeneous network equipment and resource-constrained devices. The SWAMO ontology for intelligent software agents describes the physical equipment, the process model and tasks. While the ISTAR ontology was used for selecting sensor to task allocation automatically.

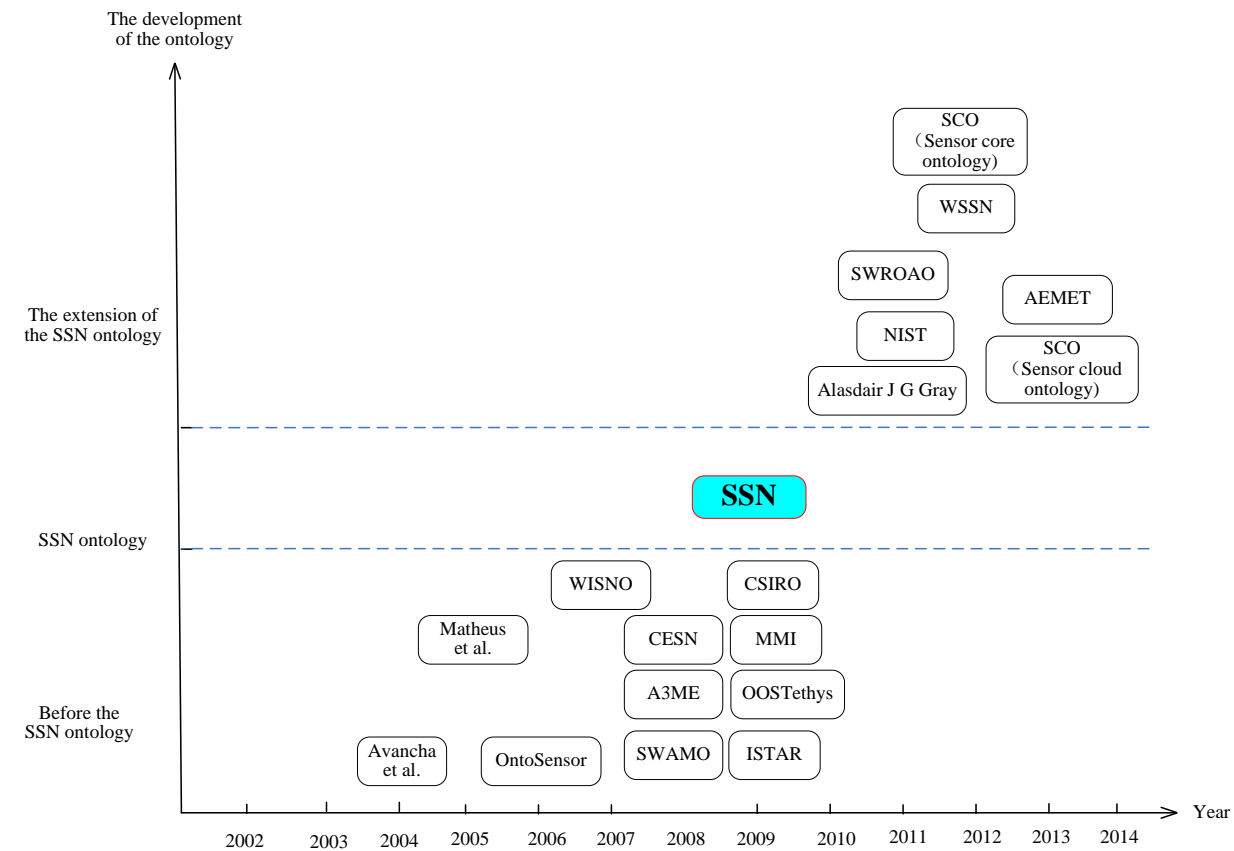

Figure 2. The Development Process of Sensor Ontology

The ontologies of the specific applications have a narrow scope of application, and they have no universality. Therefore, the general ontologies of the sensor network have been developed. The functions of the original general ontology are simple, such as Avancha [11] and WISNO [12] ontology. They are developed for providing general ontology of adaptive data processing to meet various applications. While OntoSensor [13], CESN [14], CSIRO [15] cover a wider range of ontology concepts. OntoSensor [13] ontology is a generic sensor knowledge base which is used for querying and reasoning. CESN [14] ontology can inference domain knowledge from data. CSIRO [15] is a general ontology that can describe sensor and its deployment. In all these ontologies, the combination of OntoSensor and CSIRO represent the general semantic and expression power of sensor. However, problems still exist, e.g., how to express the correct structure and the scope of the sensor ontology, how to better express process and the system composition, and how to follow a fixed modeling process and reuse the existing standards [16]. SSN ontology which develops from the CSIRO, MMI and OOTethys ontology is a general ontology and full of expressive power. It can solve the existing problems in the original ontologies.

\subsection{SSN Ontology}

To acquire the semantic interoperability of general scale, common semantic annotation framework, W3C SSN XG group [4] proposed a general, field-independent model: SSN ontology. SSN ontology provides an advanced schema to describe the sensor equipment, 
their measurement data and the relevant properties of the sensor processing. SSN ontology designs the Stimulus-Sensor-Observation (SSO) pattern to describe the relationships between the sensors, the stimulation and the observation. It can be seen from four main perspectives: the sensor perspective which focus on the sensor, the sense mode and the sense object, the observation perspective which focus on the observation data and the related metadata, the system perspective which describes systems of sensors and deployments and the feature and property perspective which concerns the sensor properties or observational properties [17]. SSN ontology builds several conceptual modules on the SSO pattern to cover key sensor concepts and includes forty one concepts and thirty nine properties.

SSN ontology is the integration and upgrade of the original ontologies. It describes more clearly because it has more detailed classification. It has a wider range of generality, great extension space. But above all, it can be extended and reused as the basis to solve more complex problems. Therefore, many applications using SSN ontology have been appeared.

SSN ontology is applied to many projects now1, such as IoT.est project [18, 19], Semantic Perception [20, 21], SECURE [22], SemSorGrid4Env [23-25], Swiss Experimen [16], SPITFIRE project [26, 27], etc.

In IoT. Est project, SSN ontology is used in the Internet of things to describe the resources, the observation and the measurement system of sensors. In Semantic Perception project, machine perception is researched. Perception ontology which reused SSN ontology is established. In the perception ontology, the key of perception explain: observation and terms of environmental knowledge is provided. SSN ontology is applied to the rescue environment In SECURE project. It describes observation data from the temperature sensors, carbon monoxide sensors, and carbon dioxide sensors, which can integrate and analyze the data, and concludes the events. In SemSorGrid4Env project, SSN ontology shows public data model of information space, and it is applied in environment monitoring system. In Swiss Experiment project, SSN ontology describes sensor meta-data and research collaborative environment and it connects with LOD cloud effectively in SPITFIRE project. In this project, it develops uniform concepts, methods and software infrastructure.

\subsection{The Extensions of SSN Ontology}

Much ontology established later is mostly the extensions of SSN ontology. The main extension directions are:

(1) Extend according to different application.

(2) Add new contents to SSN ontology to develop more general ontology.

(3) Combine with the other ontology to solve actual problem.

The three extension directions are shown as Figure 3. 


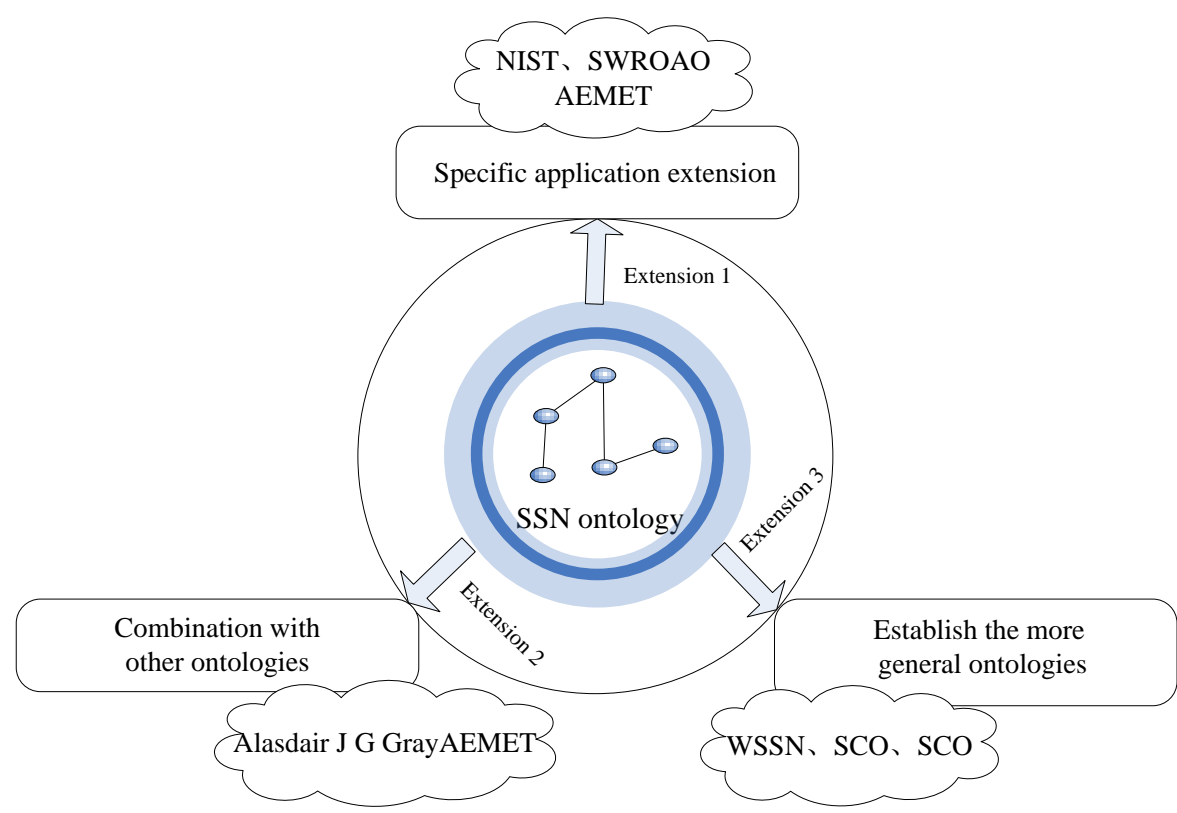

Figure 3. The Extension Direction of SSN Ontology

\section{(1) Extending According to Different Application}

SSN ontology is extended according to the specific requirements of different applications. The extended ontologies add new concepts on SSN ontology or change SSN ontology for the specific application, so that they can describe the application field more clearly.

NIST ontology [17] applies to the manufacturing industry. In NIST ontology, SSN ontology is extended according to a series of specific requirements. On the basis of SSN ontology, function, domain, additional entities, resource group, status, purpose, physical location, etc are added in NIST ontology. NIST ontology describes the detailed sensor dimension, weights, resolution and the knowledge representation standard of the operating conditions, the representation of the system ability, and the sensor network of the manufacturing environment etc.

SWROAO [28] ontology is used on atmosphere observation. The platform class of SSN ontology is extended. In SWROAO ontology, three subclasses are derived from the platform class. They are aircraft class, ground class and spacecraft class. Each class is subdivided. Aircraft class includes LEO satellite and high orbit satellite, ground class includes the ground remote sensing platform, the ground atmospheric boundary layer observation platform and the mobile ground atmospheric observation platform, and spacecraft class includes balloon, aircraft and rocket, etc. The subdivision of these specific platforms makes SWROAO ontology carry out different query or decision depending on different platforms in the applications.

AEMET ontology [29] applies to the meteorological forecast of spanish meteorological bureau. It extends from SSN ontology. In AEMET ontology, measurement ontology is related to meteorlological observation. The main conceptes reuse "ssn: Observation", "ssn: FeatureOfInterest" and "ssn: Property" of SSN ontology. But the SSN ontology concepts will be applied to a specific instance. Such as the concept "SSN: Property" is extended and added the specific properties of meteorological aspect in AEMET ontology. The concept "ssn: Sensor" is expanded to a hierarchy of types of sensors in the AEMET ontology and "ssn: Platform" is extended by "aemet: WeatherStation". 


\section{(2) Extending by Adding New Contents to SSN Ontology to Found More General Ontology}

The more general ontologies are extended on the basis of SSN ontology in order to have more extensively applicable scope.

WSSN ontology [30] enriches SSN ontology. It describes communication by a kind of new pattern and integrates new concepts relating to the wireless sensor network in SSN ontology. WSSN ontology uses a new pattern: SWSNC (Stimulus-WSNnode-Communication) ontology design pattern. This pattern treats the stimulus as the starting point of any process and the trigger of sensor or communication equipment. In the observed phenomenon, stimulation can exist as the measurable change and the unconscious stimulation of a reaction process. Stimulation can also be used as a conscious stimulus in the communication process, and it is an input communication needs. WSSN ontology adds some new concepts of wireless sensor network, such as communication, data flow and state.

SCO ontology [31] is also built by reusing and extending SSN ontology. The Sensor module in SCO ontology is the core module which is a bridge connecting to other modules. Sensor module directly reuses the Sensor module of SSN ontology, but the Stimulus class is replaced by SensorInput class. The OpeatingRestriction module is changed to the SystematicCharacter module and EnvironmentCondition class is added in the module. In addition, on the basis of SSN ontology modules, new modules such as the Component module, the Service module and the Context module, etc are added. In the Context module, three important classes are added: Space, Time and Theme. These three classes respectively show that Space, Time, and Theme of observations values by which the sensor data information can be described in more detail. SCO ontology is a major progress of SSN ontology extensions.

In order to conduct semantic description of the sensor data on the sensor cloud, SCO (Sensor Cloud Ontology) Ontology appears [32]. SCO Ontology reuses and extends SSN ontology and many classes and properties don't have to be redefined. Through the derived subclass, the platform and network and other characteristics of sensor network on the Cloud can be defined. In SCO ontology, the Network class and Platform class derived from the System class of SSN ontology, the Sensor class derived from the SensingDevice class, while the ObservedPhenomenon class derived from the Observation class, the Observation Result class derived from the SensorOutput. Using SCO ontology, the transformation from sensor data to semantic very facilitate, so that we have convenient queries on the SERAW development platform.

\section{(3) Extending by Combining with the other Ontology to Solve Actual Problem}

Because the data of Semantic Sensor Web is linked-data, the ontology combination can make the application range greatly extend. The combination of SSN ontology and the other ontologies is necessary.

Alasdair J G Gray [24] proposed SemSorGrid4Env ontology network to apply in flood emergency prediction scheme. This ontology network is composed of different ontologies. It is divided into four layers: the ontology of the specific fields, the information ontology, the upper ontologies and the external ontologies. These ontologies meet different knowledge representation requirements and they share and reuse between each other. SSN ontology reuses the DOLCE + DnS UltraLite and SWEET upper ontology. The ontologies in the flood field are the basis of SSN ontology. They also reuse the external ontology. These ontologies combine with each other and play the biggest role in order to solve the flood emergency prediction.

AEMET ontology is also formed by multiple ontologies [29]. The ontology includes four modules: sensor, time, location and measurement. The Time module reuses the OWL Time Ontology, and the location Ontology reuses part of geobuddies ontology network, 
and the measure ontology reuses the concepts of SSN Ontology. All these ontologies are used to the meteorological forecasting of the Spanish meteorological bureau. They can transform the meteorological data to linked-data and have more comprehensive description.

\subsection{Comparison and Analysis}

In order to better understand SSN ontology and the extension ontologies, comparative dimensions of ontologies are proposed in this paper. The extension direction, extension content, prototype or tool, the pattern, the language and the application domain are described. The comparative results as shown in Table 1.

In Table 1, all the ontologies are compared from several aspects. The column 2 lists the extension direction from which each sensor ontology extends. The column 3 describes the extension contents. Some sensor ontologies apply to new applications by combining with other ontologies, Some sensor ontologies extend the platform class and some design the new pattern. They all have different extension direction and add their own new classes or new concepts.

In the column 4, some tools of the ontology are proposed. Some sensor ontologies have their related system tools, but some have no. We can use them to do more research and application. For example, a prototype system named SenOntDSSW is designed for SCO ontology update and extension. The multi-agent platform JADE can integrate the WSSN ontology to generate the communication data stream, SemSorGrid4Env ontology network to apply in flood emergency prediction scheme, and SERAW is a platform for using the SCO ontology.

Table 1. The Comparison of Ontologies

\begin{tabular}{|c|c|c|c|c|c|c|c|c|}
\hline \multirow{2}{*}{$\begin{array}{l}\text { Sensor } \\
\text { ontology }\end{array}$} & \multicolumn{3}{|c|}{$\begin{array}{c}\text { Extension } \\
\text { direction }\end{array}$} & \multirow{2}{*}{ Extension content } & \multirow{2}{*}{ Prototype \& tool } & \multirow{2}{*}{ Pattern } & \multirow{2}{*}{ Language } & \multirow{2}{*}{$\begin{array}{l}\text { Application } \\
\text { domain }\end{array}$} \\
\hline & $\mathbf{1}$ & 2 & 3 & & & & & \\
\hline SSN & & & & ---- & ---- & SSO & OWL2 & general \\
\hline SWROAO & $\sqrt{ }$ & & & $\begin{array}{l}\text { The platform class is } \\
\text { extended, and several } \\
\text { subclasses are derived. }\end{array}$ & ---- & SSO & OWL-DL & $\begin{array}{l}\text { Atmospheric } \\
\text { monitoring }\end{array}$ \\
\hline NIST & $\sqrt{ }$ & & & $\begin{array}{l}\text { The concepts of the } \\
\text { manufacturing industry } \\
\text { are added in the ontology. }\end{array}$ & --- & SSO & OWL2 & manufacturing \\
\hline $\mathrm{SCO}$ & & $\sqrt{ }$ & & $\begin{array}{l}\text { In the new modules, three } \\
\text { important classes such as } \\
\text { space, time and theme are } \\
\text { added. }\end{array}$ & SenOntDSSW & SSO & OWL2 & $\begin{array}{l}\text { Weather } \\
\text { monitoring }\end{array}$ \\
\hline WSSN & & $\sqrt{ }$ & & $\begin{array}{l}\text { A new pattern which is } \\
\text { SWSNC ontology design } \\
\text { pattern is proposed. }\end{array}$ & $\begin{array}{l}\text { Multi-agent } \\
\text { platform JADE }\end{array}$ & SWSNC & OWL2 & general \\
\hline AEMET & $\sqrt{ }$ & & $\sqrt{ }$ & $\begin{array}{l}\text { New concepts are added } \\
\text { and several ontologies } \\
\text { combine with each other }\end{array}$ & Map4RDF & SSO & OWL-DL & weather forecast \\
\hline $\begin{array}{l}\text { Alasdair } \\
\text { J G gray }\end{array}$ & & & $\sqrt{ }$ & $\begin{array}{l}\text { The SemSorGrid4Env } \\
\text { ontology network is } \\
\text { proposed in which several } \\
\text { ontologies combine with } \\
\text { each other. }\end{array}$ & SemSorGrid4Env & SSO & OWL & $\begin{array}{l}\text { Flood } \\
\text { emergency } \\
\text { prediction }\end{array}$ \\
\hline
\end{tabular}




\begin{tabular}{llllll}
\hline SCO & $\sqrt{ } \quad \begin{array}{l}\text { To apply to the sensor } \\
\text { cloud environment, } \\
\text { several new classes are } \\
\text { added. }\end{array}$ & SERAW & OWL & general \\
\hline
\end{tabular}

Most of the ontologies use the SSO pattern except the WSSN ontology. Stimulus-Sensor-Observation (SSO) pattern describe the relationships between the sensors, the stimulation and the observation, but SWSNC (Stimulus-WSNnode-Communication) ontology which is designed by WSSN ontology treats the stimulus as the starting point of any process and the trigger of sensor or communication equipment.

These sensor ontologies use three languages to describe the contents: OWL2, OWL-DL and OWL. In these ontologies, SSN, WSSN, SCO are general ontologies, while the other ontologies have their specific applications.

\section{The Mapping from Stream Data to Ontology}

The core issue of semantic data integration process is to find and generate the mapping, so how to realize the mapping from the sensor stream data to the ontology is the issue which we need to solve. In this issue, we must consider an important thing that the sensor data is the stream data. The stream data is an infinite sequence of tuples and with timestamp, so in the mapping process, we must consider the time characteristic.

The mapping from sensor stream data to the ontology has two main methods:

(1) Hard coding method: The correspondence between stream data and ontology is defined in programs. Different fields and different stream data format need different coding. The stream data will be generated to RDF files according to the program coding.

(2) Mapping language method. The mapping languages can express the mappings from relational databases to RDF datasets. They describe the mapping relationships between the basic elements (such as relationship or attributes etc.,) of the relational database and the basic elements (such as concepts, attributes and relationship etc.,) of the RDFS/OWL ontology. They will provide a set of well-defined semantic primitives to describe the mapping relationships. The mapping of stream data and the ontology can be added manually or automatically by programming. Most of the mapping languages have mapping processor which can fill the data of the relational databases in the ontology automatically. The frequently-used mapping languages are: D2RQ [25, 33], $\mathrm{R}_{2} \mathrm{O}$ [34] and R2RML [16, 35, 36], RML [37], Ontop [38], $\mathrm{S}_{2} \mathrm{O}$ [23].

\subsection{The Mapping Languages}

D2RQ is a declarative language for mapping relational database schemas to RDF vocabularies and OWL ontologies. It is realized as the Jena Figure. It can pack one or more relational database to a virtual, read-only RDF graphs. Its mapping processor is D2R server which can use a customizable D2RQ mapping to map database content into the RDF format and allow the RDF data to be browsed and searched.

$\mathrm{R}_{2} \mathrm{O}$ is the declarative mapping description language based on the XML. It can describe mapping relations between the relations and the properties of the relation database and the basic elements of RDFS/OWL ontology. It can be realized by ODEMapster mapping processor.

R2RML is the mapping language announced by the W3C RDB2RDF in September 2012. It can express the mapping from RDB to RDF by using a form of one or more triples mapping elements. R2RML mappings are themselves RDF graphs and written down in Turtle syntax. R2RML processor will offer a virtual SPARQL endpoint over the mapped relational data, or generate RDF files, or offer a Linked Data interface. In reference [39], R2RML processor prototype is designed. It can transform RDB to RDF 
automatically. Morph-RDB is an RDB2RDF engine which follows the R2RML specification. It can generate RDF data from a relational database according to the R2RML mapping descriptions.

The RDF Mapping language (RML) is a generic mapping language defined to express customized mapping rules from heterogeneous data structures and serializations to the RDF data model. RML is defined as a superset of the W3C-standardized mapping language, R2RML, that maps data in relational databases to the RDF data model. RML extends R2RML's applicability and broaden R2RML's scope to define mappings of data in other formats too [37]. With RML, you can define the rules to map any heterogeneous data such as DB, CSV, XML and JSON etc to the RDF data model. A prototype RML processor is implemented in Java based on the mapping-driven model.

Ontop is a platform to query databases as Virtual RDF Graphs using SPARQL. It's extremely fast and is packed with features. It concludes two components: Quest and ontopPro. Quest is the SPARQL engine that comes with ontop, and ontopPro is a powerful mapping editor to allow you to define data sources for your ontologies and to map them using a powerful and intuitive mapping language. The data sources conclude a variety of data formats such as DB, CSV, XML, XSL etc.

$\mathrm{S}_{2} \mathrm{O}$ is the extension of $\mathrm{R}_{2} \mathrm{O}$ mapping language, and it mainly shows the mapping relationship from the sensor stream data to the ontology. $\mathrm{S}_{2} \mathrm{O}$ mapping documents describe how to convert the stream data source to the ontology. It is the extensional version of $\mathrm{R}_{2} \mathrm{O}$. In order to support stream data, $\mathrm{S}_{2} \mathrm{O}$ can describe the stream data model. On the basis of $\mathrm{R}_{2} \mathrm{O}$, new correspondence relationships are added: streamschema-desc. The streamType property is increased to describe a kind of data acquisition stream. The timestamp-desc element is increased to declare the stream timestamp property. The other classes and properties defined by $\mathrm{R}_{2} \mathrm{O}$ are all suitable for stream pattern.

\subsection{Comparison and Analysis}

The comparison of mapping languages is shown as the Table 2. The line 2 describe if the mapping languages have the timestamp character. $\mathrm{S}_{2} \mathrm{O}$ is a mapping language designed for sensor data specially, but the other mapping languages are general mapping languages.

These mapping languages have different representation languages. D2RQ mapping file is Jena Figure, $\mathrm{R}_{2} \mathrm{O}$ and $\mathrm{S}_{2} \mathrm{O}$ are an XML file, while R2RML is the .ttl document written by the turtle syntax and RML files are one or more triples maps.

They all have mapping processors which can realize transformation automatically. D2RQ server uses a customizable D2RQ mapping to map relational database contents into RDF. The mapping processor of $\mathrm{R}_{2} \mathrm{O}$ is ODEMapster. Morph-RDB is the extension of ODEMapster. It can generate RDF data from a relational database according to the R2RML mapping descriptions. RMLProcessor is the proessor for RML in Java based on DB2Triples. The processor of Ontop is ontopPro, while $\mathrm{S}_{2} \mathrm{O}$ 's processor is an extension of the ODEMapster processor, it can realize the mapping from the stream data to RDF files.

Table 2. The Comparison of the Mapping Languages

\begin{tabular}{|c|c|c|c|c|c|c|}
\hline Mapping & D2RQ & $\mathrm{R}_{2} \mathrm{O}$ & R2RML & RML & Ontop & $\mathrm{S}_{2} \mathrm{O}$ \\
\hline Timestamp & no & no & no & no & no & yes \\
\hline $\begin{array}{l}\text { Representation } \\
\text { language }\end{array}$ & $\begin{array}{l}\text { Jena } \\
\text { Figure }\end{array}$ & XML & $\begin{array}{l}\text {.ttl documents } \\
\text { using turtle } \\
\text { grammar }\end{array}$ & $\begin{array}{l}\text { One or more } \\
\text { Triples Maps }\end{array}$ & ---- & XML \\
\hline
\end{tabular}




\begin{tabular}{|c|c|c|c|c|c|c|}
\hline $\begin{array}{l}\text { Mapping } \\
\text { processor }\end{array}$ & $\begin{array}{l}\mathrm{D} 2 \mathrm{R} \\
\text { server }\end{array}$ & $\begin{array}{l}\text { ODEMapste } \\
\mathrm{r}\end{array}$ & $\begin{array}{l}\text { R2RML } \\
\text { Processor } \\
\text { prototype[39] } \\
\text { morph-RDB }\end{array}$ & $\begin{array}{l}\text { RMLProcesso } \\
\mathrm{r}\end{array}$ & ontopPro & $\begin{array}{l}\text { an extension } \\
\text { of the } \\
\text { ODEMapste } \\
\mathrm{r} \text { processor }\end{array}$ \\
\hline Data resources & $\mathrm{RDB}$ & $\mathrm{RDB}$ & $\mathrm{RDB}$ & $\begin{array}{l}\text { DB, CSV, } \\
\text { TSV } \\
\text { XML, JSON }\end{array}$ & $\begin{array}{l}\text { DB, CSV, } \\
\text { XML, } \\
\text { XSL }\end{array}$ & Stream data \\
\hline $\begin{array}{l}\text { Application } \\
\text { case }\end{array}$ & [33] & [34] & $\begin{array}{l}\text { morph-GFT } \\
\text { morph-stream } \\
\mathrm{s}\end{array}$ & {$[40,41]$} & $\begin{array}{l}{[38]} \\
\text { The } \\
\text { Optique } \\
\text { project }\end{array}$ & {$[23,42]$} \\
\hline website & $\begin{array}{l}\text { http://d2rq. } \\
\text { org/d2rq-la } \\
\text { nguage }\end{array}$ & $\begin{array}{l}\text { http://www. } \\
\text { r2o.net.nz/ }\end{array}$ & $\begin{array}{l}\text { http://www.w } \\
\text { 3.org/TR/r2rm } \\
\text { 1/ }\end{array}$ & http://rml.io/ & $\begin{array}{l}\text { http://onto } \\
\text { p.inf.unibz } \\
\text {.it }\end{array}$ & ---- \\
\hline
\end{tabular}

D2RQ, $\mathrm{R}_{2} \mathrm{O}, \mathrm{R} 2 \mathrm{RML}$ can transform the relational database to RDF or OWL files, while RML and Ontop can transform multiple data formats such as DB, CSV, XML, JASON etc. $\mathrm{S}_{2} \mathrm{O}$ mapping language applies to semantic sensor network, and it can convert the stream data to the RDF file.

All these mapping languages have some application cases which are showed in the table. Among them, morph-streams are an ontology-based data access system that applies to the sensor network and queries the sensor data. It is mapped using R2RML language. $\mathrm{S}_{2} \mathrm{O}$ mapping language special applies to the sensor network. From the websites in the table, we can see the detailed content and the grammar of every mapping language.

\section{Query Tools and Methods}

As above mentioned, sensor data stream are an unbounded sequence values, and each value has a timestamp to represent the value of happened time. People often are interest in data values of the latest stream data, while they don't concerned about the previous tuples. Traditional SQL query adapts to the storage data, while the stream query requires continuous long-term queries. In order to realize the stream query, a few continuous query languages based on SQL appears. Such as CQL [43], SNEEql [44], Esper EPL [45], etc. These languages have ability to deal with time constraints and the infinite characteristics of stream. They can put forward the concept of the window and use traditional relational operators to execute continuous query [46].

\subsection{The Query Languages}

SPARQL language [47] is a kind of query language developed for RDF. It can easily query in RDF files. In the Semantic Sensor Web, we need to query the continuous stream data, but SPARQL language can't effectively work. SPARQL language must be extended to achieve the goal of the continuous query of the stream data.

The earliest query languages are T-SPARQL [48], TA-SPARQL [49, 50], EP-SPARQL [51], C-SPARQL [52-54], and stSPARQL [55, 56], etc. They all use RDF stream as the data model. The tuples of RDF streams are annotated by timestamp.

T-SPARQL is a temporal extension of the SPARQL query language for RDF graphs. It is the RDF database model based on time, and this model uses the triple timestamp with time elements. The query language came from a time query language TSQL2 and inherited part time expression and user friendliness of TSQL2. T-SPARQL is the extension of SPARQL query language in terms of time data types, functions and operators, and it enhances properties of TSQL2. In the coming research, T-SPARQL 
query engine is devoted to develop to make the queries of the time RDF graphs more convenient.

Time-annotated RDF (TA-RDF) is an extension of the RDF model where resources are optionally annotated with a time value. Along with TA-RDF, Time-annotated SPARQL (TA-SPARQL) is designed to express the one-time time-dependent queries common when handling streams and time-varying data. TA-SPARQL goes beyond other query languages for streams in RDF presented previously and base on sliding windows, and allows to exploit the richer temporal relationships represented by the semantic model [49]. The TA-RDF and TA-SPARQL have been implemented in a prototype service, integrated with the NCSA Tupelo semantic middleware which can make the stream data and other data integrate and perform unified query.

EP-SPARQL is the SPARQL extension query language of event processing. It can query complex events and conduct stream reasoning. EP-SPARQL is put forward in order to be able to query based on stream. It considers the time situation asserted by triples and specifies the complex events through the time position of real-time stream, and use background ontology for stream reasoning.

C-SPARQL is the expansion of SPARQL language. It can realize continuous query of RDF data stream. C-SPARQL query puts forward the concept of window. It can query the latest tuples of data stream and observe the continuous data streams. C-SPARQL manages RDF stream tuples locally in data model, at the same time converts the unbounded stream data to a bounded sequence using time window, then the standard operators can be used. Okure, A.S. [57] proposes architecture which can query more OWL ontology stream which carry the latest real-time time stream reasoning.

The stRDF data model and the stSPARQL query language are developed by Manolis Koubarakis [55, 56]. stRDF adds the elements of time and space in RDF triples, so it can express spatio-temporal data. stSPARQL is an expansion of SPARQL, and it can query stRDF data. stSPARQL provides a new type of variables: Spatial variables, temporal variables. It can query valid time of triples. In SemsorGrid4Env project, Kostis Kyzirakos Strabon [56] proposed Strabon, which is a fully implemented semantic geospatial database system. Strabon can be used to store linked geospatial data expressed in stRDF and query them using stSPARQL.

The query premise of the above query languages is the existence of the RDF file, namely, the query object is RDF files. But the query based on ontology has another form that is RDF files are not generated. From the user's perspective, SPARQL query language is used to query, and virtual query statements are generated. The SPARQL query languages are transformed to SNEEql query statements aiming at stream data through R2RML or S2O mapping language, and the stream data stored in the relational database can be queried directly. The query results are transformed using the mapping language, so query results are acquired $[4,5,25]$. In this method, SPARQLStream is the using query language [46].

SPARQL $_{\text {Stream }}$ is a language that extends C-SPARQL and SNEEql for continuous query processing over streaming data. They all are the idea based on RDF stream, and

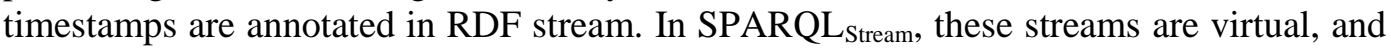
they generate query results relying on original data stream. In order to convert SPARQL $L_{\text {Stream }}$ queries expressed by ontology to the queries according to data sources, a set of mapping must be specified. SNEEql queries that conduct against the data source can be acquired by the mapping, which can use SQL statements to query, and query results can be obtained. The Morph-streams module for SPARQL ${ }_{\text {Stream }}$ is a java library that enables the execution of SPARQL $\mathrm{S}_{\text {Stream }}$ queries, using different underlying DSMS or CEP. This tool allows posing SPARQL ${ }_{\text {Stream }}$ queries to an existing data source using R2RML mappings. 


\subsection{Comparison and Analysis}

The comparison of query languages is shown as the Table 3.

Table 3. The Comparison of the Query Languages

\begin{tabular}{|c|c|c|c|c|c|c|}
\hline & T-SPARQL & TA-SPARQL & EP-SPARQL & C-SPARQL & stSPARQL & SPARQL $_{\text {stream }}$ \\
\hline Abbreviation & $\begin{array}{l}\text { Temporal-SPA } \\
\text { RQL }\end{array}$ & $\begin{array}{l}\text { Time-Annotated } \\
\text { SPARQL }\end{array}$ & $\begin{array}{l}\text { Event } \\
\text { Processing-SPA } \\
\text { RQL }\end{array}$ & $\begin{array}{l}\text { Continues-SPA } \\
\text { RQL }\end{array}$ & $\begin{array}{l}\text { Spatial and } \\
\text { temporal-SPA } \\
\text { RQL }\end{array}$ & SPARQL $_{\text {stream }}$ \\
\hline $\begin{array}{l}\text { Time windou } \\
\text { query }\end{array}$ & yes & yes & yes & yes & yes & yes \\
\hline $\begin{array}{l}\text { Virtual RDF } \\
\text { file }\end{array}$ & no & no & no & no & no & yes \\
\hline RDF model & $\mathrm{tRDF}$ & TA-RDF & RDF stream & $\mathrm{RDF}$ & stRDF & $\begin{array}{l}\text { Virtual } \\
\text { stream }\end{array}$ \\
\hline derived from & TSQL2 & T-SPARQL & SPARQL & SPARQL & SPARQL & $\begin{array}{l}\text { C-SPARQL } \\
\text { SNEEq1 }\end{array}$ \\
\hline RDF triple & $<s, p, o \mid T>$ & $<s\left[t_{s}\right], p\left[t_{p}\right], o\left[t_{o}\right]>$ & $\ll s, p, o>, t, t^{\prime}>$ & $<<s, p, o>, T>$ & $<s, p, o, T>$ & $<<s, p, o>, T>$ \\
\hline Application & $\begin{array}{l}\text { The tSPARQL } \\
\text { query engine }\end{array}$ & $\begin{array}{l}\text { NCSA Tupelo } \\
\text { semantic } \\
\text { middleware }\end{array}$ & ETALIS & $\begin{array}{l}\text { C-SPARQL } \\
\text { engine }\end{array}$ & Strabon & Morph-stream \\
\hline $\begin{array}{l}\text { Extension } \\
\text { directions }\end{array}$ & $\begin{array}{l}\text { Time-dependen } \\
\text { t queries }\end{array}$ & $\begin{array}{l}\text { Time-dependent } \\
\text { queries }\end{array}$ & Event processing & $\begin{array}{l}\text { Support } \\
\text { windows } \\
\text { aggregation } \\
\text { operations }\end{array}$ & $\begin{array}{l}\text { Query spatial, } \\
\text { temporal data }\end{array}$ & Virtual query \\
\hline
\end{tabular}

In Table 3, the query languages are compared from several aspects. All the query languages apply to the sensor network and they all describe the time characteristic of the sensor data from different aspects. The line 2 lists the abbreviations of every query languages and the line 3 shows all the query language use the time window for the query. In the query process, all the query languages make the actual RDF files as the query object except the SPARQL $L_{\text {Stream }}$ query language. It just generates a virtual RDF query and RDF file does not exist.

The line 5 gives the RDF models of the query language which are different. The entire RDF models are the extension of RDF, and they provide convenience for the stream data query. The RDF triples are showed in the Table. They all add the time element in the triples in order to represent the stream data more accurately. The line 6 shows where the query languages derived from.

All query languages have their application system. The tSPARQL query engine can facilitate the execution of T-SPARQL queries on temporal RDF graphs, and C-SPARQL engine makes reason over RDF stream easy. An implementation of TA-SPARQL based on Tupelo semantic middleware is implementation. ETALIS is an open source system for complex event processing with ETALIS language for Events and EP-SPARQL. Strabon can query linked geospatial data using stSPARQL and Morph-stream allows executing SPARQL $_{\text {stream }}$ queries to an existing data source using R2RML mappings.

All query languages are the extension of the SPARQL language. T-SPARQL and TA-SPARQL carry out the time-dependent queries, while EP-SPARQL applies to the event processing. C-SPARQL support windows and aggregation operations and stSPARQL query spatial and temporal data, while SPARQL $\mathrm{L}_{\text {stream }}$ executes virtual query. 


\section{Conclusion and Future Work}

In this paper, the current research progress of Semantic Sensor Web was summarized. The introductions of this paper are expected to promote the focus and research on this emerging technology by the related scholars. We expect that the future development directions of Semantic Sensor Web include the following aspects [58, 59].

(1) Researching in the cloud platform

In the real environment, the data size of sensor network is very huge. The integration between sensor network and cloud computing is one of the current development trend. The combination of semantic Web, big data technology and cloud computing technology can conduct distributed storage for ontology instance knowledge. The combination can explore how to improve the interaction of heterogeneous sensor network data, enhance the intelligent ways and means of the sensor network application system, and provide new idea and theory basis for building intelligent and efficient decision-making application system $[60,61]$.

(2) Linking with the open data source

The data in Semantic Sensor Web are the linked data. The link scope is broader, the search scope is bigger. Therefore, that the domain ontology connects with the existing linked open data sources such as linked geodata and DBpedia is needed. The combination can make the deducibility semantic description of sensor information and a extensible data model will be obtained. The model can be used to conduct query in sensor data and context connection data. SPITFIRE FP7 project which was developed by Myriam Leggieri [27] devotes to this research.

(3) Combining with social network

Society is a big sensor community made up of all human, and each person is an intelligent sensor. These sensors move autonomously in the social and the natural environment. They can perceive, interpret, and integrate information, and can conduct the mutual exchange of information through social networks. Therefore, the combination of the Semantic Sensor Web and social network is a development trend of the future [62-65].

(4) Combining with artificial intelligence

Semantic Sensor Web can also combine with artificial intelligence. The combination will establish question-answering system for the application query of Semantic Sensor Web, understand and recognize the natural language, and conduct intelligent query. All these will be the future research directions.

(5) The visualization of Semantic Sensor Web

The visualization research of the query results or the real query of Semantic Sensor Web is also a research direction in the future. Query results are displayed on the electronic map. When click the place on the map, you can get the specific query information or the implicit information. The data visualization technology can also used to automatically map data into charts, and enables users to easily find the implicit information in the sensor network $[66,67]$.

\section{Acknowledgements}

This work is supported by Science Foundation of Hebei Province (No. F2013208107), National Natural Science Foundation of China (No.51271033), Science Foundation Project of Hebei Education Department (No. QN20131138). It is also supported by Hebei University of Science and Technology Research Foundation for the Doctoral Program (No. QD201214).

\section{References}

[1] A. Sheth, C. Henson and S. S. Sahoo, "Semantic sensor web", Internet Computing, IEEE, vol. 12, (2008), pp. 78-83. 
[2] M. Compton, C. A. Henson, H. Neuhaus, L. Lefort and A. P. Sheth, "A Survey of the Semantic Specification of Sensors", in SSN, (2009), pp. 17-32.

[3] D. J. Russomanno, C. Kothari and O. Thomas, "Sensor ontologies: from shallow to deep models", in System Theory, 2005. SSST"05. Proceedings of the Thirty-Seventh Southeastern Symposium on, (2005), pp. 107-112.

[4] M. Compton, P. Barnaghi, L. Bermudez, R. García-Castro, O. Corcho, S. Cox, et al., "The SSN ontology of the W3C semantic sensor network incubator group", Web Semantics: Science, Services and Agents on the World Wide Web, vol. 17, (2012), pp. 25-32.

[5] C. J. Matheus, D. Tribble, M. M. Kokar, M. G. Ceruti and S. C. McGirr, "Towards a formal pedigree ontology for level-one sensor fusion", DTIC Document, (2006).

[6] A. Herzog, D. Jacobi and A. Buchmann, "A3ME-an Agent-Based middleware approach for mixed mode environments”, in Mobile Ubiquitous Computing, Systems, Services and Technologies, UBICOMM'08. The Second International Conference on, (2008), pp. 191-196.

[7] K. J. Witt, J. Stanley, D. Smithbauer, D. Mandl, V. Ly, A. Underbrink, et al., "Enabling sensor webs by utilizing SWAMO for autonomous operations", in NASA Earth Science Technology Conference (ESTC2008), (2008).

[8] C. Rueda, N. Galbraith, R. A. Morris, L. E. Bermudez, R. A. Arko and J. Graybeal, "The MMI Device Ontology: Enabling Sensor Integration", in AGU Fall Meeting Abstracts, (2010), pp. 08.

[9] E. Bridger, L. Bermudez, M. Maskey, C. Rueda, B. Babin and R. Blair, "OOSTethys-Open Source Software for the Global Earth Observing Systems of Systems", in AGU Fall Meeting Abstracts, (2009), pp. 1065.

[10] M. Gomez, A. Preece, M. P. Johnson, G. De Mel, W. Vasconcelos, C. Gibson, et al., "An ontology-centric approach to sensor-mission assignment", in Knowledge Engineering: Practice and Patterns, ed: Springer, (2008), pp. 347-363.

[11] S. Avancha, C. Patel and A. Joshi, "Ontology-Driven Adaptive Sensor Networks", in MobiQuitous, (2004), pp. 194-202.

[12] Y. Hu, Z. Wu and M. Guo, "Ontology driven adaptive data processing in wireless sensor networks", in Proceedings of the 2nd international conference on Scalable information systems, (2007), pp. 46.

[13] D. J. Russomanno, C. R. Kothari and O. A. Thomas, "Building a Sensor Ontology: A Practical Approach Leveraging ISO and OGC Models", in IC-AI, (2005), pp. 637-643.

[14] M. Calder, R. A. Morris and F. Peri, "Machine reasoning about anomalous sensor data", Ecological Informatics, vol. 5, (2010), pp. 9-18.

[15] M. Compton, H. Neuhaus, K. Taylor and K.-N. Tran, "Reasoning about Sensors and Compositions", in SSN, (2009), pp. 33-48.

[16] J.-P. Calbimonte, H. Jeung, O. Corcho and K. Aberer, "Semantic sensor data search in a large-scale federated sensor network", (2011).

[17] C. Schlenoff, T. Hong, C. Liu, R. Eastman and S. Foufou, "A literature review of sensor ontologies for manufacturing applications", in Robotic and Sensors Environments (ROSE), 2013 IEEE International Symposium on, (2013), pp. 96-101.

[18] W. Wang, S. De, G. Cassar and K. Moessner, "Knowledge representation in the internet of things: semantic modelling and its applications", Automatika-Journal for Control, Measurement, Electronics, Computing and Communications, vol. 54, (2013).

[19] P. Barnaghi, W. Wang, C. Henson and K. Taylor, "Semantics for the Internet of Things: early progress and back to the future", International Journal on Semantic Web and Information Systems (IJSWIS), vol. 8, (2012), pp. 1-21.

[20] C. Henson, A. Sheth and K. Thirunarayan, "Semantic perception: Converting sensory observations to abstractions", Internet Computing, IEEE, vol. 16, (2012), pp. 26-34.

[21] C. Henson, K. Thirunarayan and A. Sheth, "An ontological approach to focusing attention and enhancing machine perception on the Web", Applied Ontology, vol. 6, (2011), pp. 345-376.

[22] P. Desai, C. Henson, P. Anatharam and A. Sheth, "SECURE: Semantics Empowered resCUe Environment (Demonstration Paper)", in 4th International Workshop on Semantic Sensor Networks 2011 (SSN 2011), (2011), pp. 115-118.

[23] J.-P. Calbimonte, O. Corcho and A. J. Gray, "Enabling ontology-based access to streaming data sources", in The Semantic Web-ISWC 2010, ed: Springer, (2010), pp. 96-111.

[24] A. J. Gray, R. García-Castro, K. Kyzirakos, M. Karpathiotakis, J.-P. Calbimonte, K. Page, et al., "A semantically enabled service architecture for mashups over streaming and stored data", in The Semanic Web: Research and Applications, ed: Springer, (2011), pp. 300-314.

[25] K. R. Page, A. J. Frazer, B. J. Nagel, D. C. De Roure, and K. Martinez, "Semantic access to sensor observations through web APIs", in Semantic Computing (ICSC), 2011 Fifth IEEE International Conference on, (2011), pp. 336-343.

[26] D. Pfisterer, K. Romer, D. Bimschas, O. Kleine, R. Mietz, C. Truong, et al., "SPITFIRE: toward a semantic web of things", Communications Magazine, IEEE, vol. 49, (2011), pp. 40-48.

[27] M. Leggieri, A. Passant and M. Hauswirth, "in Context-Sensing: LOD augmented sensor data", in Proceedings of the 10th International Semantic Web Conference (ISWC 2011), (2011). 
[28] C. Wang, N. Chen, C. Hu, S. Yan and W. Wang, "A general Sensor Web Resource Ontology for atmospheric observation", in Geoscience and Remote Sensing Symposium (IGARSS), 2011 IEEE International, (2011), pp. 3436-3439.

[29] G. Atemezing, O. Corcho, D. Garijo, J. Mora, M. Poveda-Villalón, P. Rozas, et al., "Transforming meteorological data into linked data", Semantic Web, vol. 4, (2013), pp. 285-290.

[30] R. Bendadouche, C. Roussey, G. De Sousa, J.-P. Chanet and K. M. Hou, "Extension of the semantic sensor network ontology for wireless sensor networks: the stimulus-wsnnode-communication pattern", in 5th International Workshop on Semantic Sensor Networks in conjunction with the 11th International Semantic Web Conference (ISWC), (2012).

[31] Y. Shi, G. Li, X. Zhou and X. Zhang, "Sensor Ontology Building in Semantic Sensor Web", in Internet of Things, ed: Springer, (2012), pp. 277-284.

[32] H. Müller, L. Cabral, A. Morshed and Y. Shu, "From RESTful to SPARQL: A Case Study on Generating Semantic Sensor Data", in SSN@ ISWC, (2013), pp. 51-66.

[33] L. Hu, J. Jiang, J. Zhou, K. Zhao, L. Chen and H. Lu, "Environment Observation System based on Semantics in the Internet of Things", Journal of Networks, vol. 8, (2013), pp. 2721-2727.

[34] J. Barrasa Rodríguez, Ó. Corcho and A. Gómez-Pérez, "R2O, an extensible and semantically based database-to-ontology mapping language", ( 2004).

[35] J.-P. Calbimonte, "Ontology-based access to sensor data streams", Informatica, (2013).

[36] S. Das, S. Sundara and R. Cyganiak, "R2RML: RDB to RDF mapping language", World Wide Web Consortium, (2012).

[37] A. Dimou, M. Vander Sande, P. Colpaert, R. Verborgh, E. Mannens, and R. Van de Walle, "RML: a generic language for integrated RDF mappings of heterogeneous data", in Proceedings of the 7th Workshop on Linked Data on the Web (LDOW2014), Seoul, Korea, (2014).

[38] M. Rodríguez-Muro, R. Kontchakov and M. Zakharyaschev, "Ontology-based data access: Ontop of databases", in The Semantic Web-ISWC 2013, ed: Springer, (2013), pp. 558-573.

[39] S. Zhou, Z. Xu, Y. Ni and H. Zhang, "R2RML Processor for Materializing RDF View of Relational Data: Algorithms and Experiments", in Web Information System and Application Conference (WISA), 2013 10th, (2013).

[40] A. Dimou, M. V. Sande, J. Slepicka, P. Szekely, E. Mannens, C. Knoblock and R. V. D. Walle, "Mapping hierarchical sources into RDF using the RML mapping language", in Semantic Computing (ICSC), IEEE International Conference on, (2014).

[41] A. Dimou, M. Vander Sande, P. Colpaert, L. De Vocht, R. Verborgh, E. Mannens and R. Van de Walle, "Extraction and Semantic Annotation of Workshop Proceedings in HTML using RML", in Semantic Publishing Challenge of the 11th Extended Semantic Web Conference, (2014).

[42] J. P. Calbimonte, O. Corcho and A. J. Gray, "Ontology-based access to streaming data", in Poster at the 7th Extended Semantic Web Conference (ESWC2010), Hersonissos, Greece, (2010).

[43] A. Arasu, S. Babu, and J. Widom, "CQL: A language for continuous queries over streams and relations", Database Programming Languages, Springer Berlin Heidelberg, vol. 2921, (2004), pp. 1-19.

[44] I. Galpin, C. Y. Brenninkmeijer, F. Jabeen, A. A. Fernandes and N. W. Paton, "An architecture for query optimization in sensor networks", in Data Engineering, 2008. ICDE 2008. IEEE 24th International Conference on, (2008).

[45] M. Döhring, L. Karg, E. Godehardt and B. Zimmermann, "The Convergence of Workflows, Business Rules and Complex Events", in ICEIS'10, (2010).

[46] J. P. Calbimonte, H. Jeung, Ó. Corcho and K. Aberer, "Enabling query technologies for the semantic sensor web", International Journal on Semantic Web and Information Systems, vol. 8, (2012), pp. 43-63.

[47] E. Prud'Hommeaux and A. Seaborne, "SPARQL query language for RDF", W3C recommendation, vol. 15, (2008).

[48] F. Grandi, "T-SPARQL: A TSQL2-like Temporal Query Language for RDF”, in ADBIS (Local Proceedings), (2010), pp. 21-30.

[49] A. Rodriguez, R. McGrath, Y. Liu, J. Myers and I. Urbana-Champaign, "Semantic management of streaming data", Proc. Semantic Sensor Networks, vol. 80, (2009), pp. 80-95.

[50] A. Analyti and I. Pachoulakis, "A Survey on Models and Query Languages for Temporally Annotated RDF", vol. 3, (2008), pp. 28-35.

[51] D. Anicic, P. Fodor, S. Rudolph and N. Stojanovic, "EP-SPARQL: a unified language for event processing and stream reasoning", in Proceedings of the 20th international conference on World wide web, (2011).

[52] D. F. Barbieri, D. Braga, S. Ceri, E. D. Valle, and M. Grossniklaus, "Querying rdf streams with c-sparql," ACM SIGMOD Record, vol. 39, (2010), pp. 20-26.

[53] D. F. Barbieri, D. Braga, S. Ceri and M. Grossniklaus, "An execution environment for C-SPARQL queries", in Proceedings of the 13th International Conference on Extending Database Technology, (2010).

[54] D. F. Barbieri, D. Braga, S. Ceri, E. D. VALLE, and M. Grossniklaus, "C-sparql: a continuous query language for rdf data streams", International Journal of Semantic Computing, vol. 4, (2010), pp. 3-25. 
[55] M. Koubarakis and K. Kyzirakos, "Modeling and querying metadata in the semantic sensor web: The model stRDF and the query language stSPARQL", the semantic web: research and applications. Springer Berlin Heidelberg, vol. 6088, (2010), pp. 425-439.

[56] K. Kyzirakos, M. Karpathiotakis and M. Koubarakis, "Developing Registries for the Semantic Sensor Web using stRDF and stSPARQL", in International Workshop on Semantic Sensor Networks, (2010).

[57] A. S. Okure and J. Z. Pan, "Querying EL Ontology Stream with C-SPARQL", in Advanced Computer Science Applications and Technologies (ACSAT), International Conference on, (2013).

[58] C. Ji, J. Liu and X. Wang, "A Review for Semantic Sensor Web Research and Applications", Advanced Science and Technology Letters, vol. 48, (2014), pp. 31-36.

[59] P. Barnaghi, W. Wang, L. Dong and C. Wang, "A Linked-Data Model for Semantic Sensor Streams", in Green Computing and Communications (GreenCom), IEEE and Internet of Things (iThings/CPSCom), IEEE International Conference on and IEEE Cyber, Physical and Social Computing, (2013).

[60] R. Dautov, D. Kourtesis, I. Paraskakis and M. Stannett, "Addressing self-management in cloud platforms: a semantic sensor web approach", in Proceedings of the 2013 international workshop on Hot topics in cloud services, (2013).

[61] A. Zaslavsky, C. Perera and D. Georgakopoulos, "Sensing as a service and big data", arXiv preprint arXiv: 1301.0159, (2013).

[62] D. Adams, "Citizen sensing, social signals, and enriching human experience", (2009).

[63] A. Sheth, "Computing for human experience: Semantics-empowered sensors, services, and social computing on the ubiquitous web", Internet Computing, IEEE, vol. 14, (2010), pp. 88-91.

[64] D. P. Deng, G. S. Mai, T. R. Chuang, R. Lemmens and K. T. Shao, "Social Web Meets Sensor Web: From User-Generated Content to Linked Crowdsourced Observation Data", the Semantic Web - ISWC Lecture Notes in Computer Science, Springer Berlin Heidelberg, vol. 7031, (2011), pp 257-272.

[65] J. Breslin, S. Decker, M. Hauswirth, G. Hynes, D. L. Phuoc, A. Passant, et al., "Integrating social networks and sensor networks", W3C Workshop on the Future of Social Networking, (2009).

[66] J. Howse, G. Stapleton, K. Taylor and P. Chapman, "Visualizing ontologies: a case study," the Semantic Web-ISWC 2011. Springer Berlin Heidelberg, vol. 7031, (2011), pp. 257-272.

[67] H. Patni, S. Sahoo, C. Henson and A. Sheth, "Provenance aware linked sensor data", in Proceedings of the second workshop on trust and privacy on the social and semantic web, (2010).

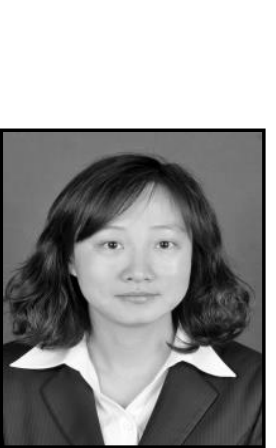

\section{Authors}

Xiang Wang, she was born in 1978, received the $\mathrm{PhD}$ degree in mechanical design and theory from University of Yanshan Qinhuangdao of China in 2012, and the Master degree in computer application from Hebei University of Technology Tianjin of China in 2004. Now, she is a lecturer in the School of Information Science and Engineering at Hebei University of Science and Technology, China. Her main research interests include Semantic Web and big data integration.

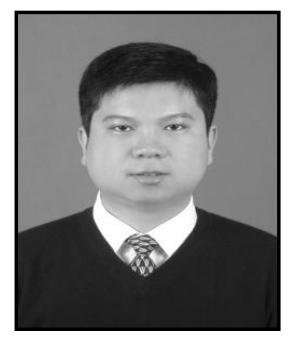

Xiaoming Zhang, she was born in 1975, received the $\mathrm{PhD}$ degree in computer application from University of Science and Technology Beijing of China in 2009, and the Master degree in computer application from Hebei University of China in 2002. Now, he is an associate professor in the School of Information Science and Engineering at Hebei University of Science and Technology, China. His main research interests include Semantic Web and domain-specific information integration.

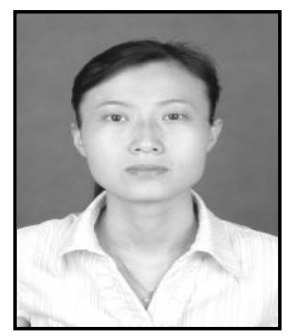

Mei Li, she was born in 1980, she obtained Master's degree in 2007 in Computer Application and Technology from Henan University of Science and Technology, China. Now she is working with Hebei University of Science and Technology, Shijiazhuang, China. Her main research area is data mining and other related areas. Email: limei@ @ebust.edu.cn 